\title{
Gestión por procesos para la Competitividad de una Mype del Sector Hotelero Frente al Covid-19
}

\section{Processes Management for Competitiveness of a SME Company in the Hotel Sector to Face of Covid-19}

\author{
Odar R. Florián Castillo, MBA. ${ }^{1}$, Angela Victoria Lozada Silva, Ing. ${ }^{2}$ and Luisa Fernanda Zavaleta Alfaro, Ing. ${ }^{2}$ \\ ${ }^{1}$ Universidad Privada del Norte, Perú, odar.florian@upn.edu.pe \\ ${ }^{2}$ Ingeniería Empresarial, Universidad Privada del Norte, Perú, angelalozada199704@gmail.com, lzavaletaalfaro@gmail.com
}

\begin{abstract}
The objective of the research was to design a process management model for competitiveness in an MSE in the Hotel Sector against COVID-19.

The methodology used is prospective-descriptive, a questionnaire reliable (Cronbach's Alpha with 0.736) and validated in the opinion of experts was applied to 27 clients (convenience sampling) and collaborators. The company complies with what is necessary, however, they do not exceed the expectations of their clients, due to the absence of good practices in process management.

A new process management model was developed, analyzing the hotel internally and externally, the activities of the reservation process were detailed, their characterization and indicator sheet for the process under evaluation.

Concluding that the company is strong internally with 2.64 in the EFI matrix and 2.67 in the EFE matrix, which means a favorable environment, therefore 12 strategies were established in the SWOT matrix. The as is diagram was restructured, generating a reservation process that contains digital activities, the implementation costs were estimated with a total of S / 9'204 and the social and environmental impact matrix was prepared in order to achieve greater sustainability and competitiveness in the organization.
\end{abstract}

Keywords-Process management, competitiveness, reservation process, sustainability, Covid-19.

Resumen-La investigación tuvo como objetivo diseñar un modelo de gestión por procesos para la competitividad en una MYPE del Sector Hotelero frente al COVID-19.

La metodología utilizada es prospectiva-descriptiva, se aplicó un cuestionario confiable (Alfa de Cronbach con 0.736) y validado por juicio de expertos a 27 clientes (muestreo por conveniencia) y a los colaboradores. La empresa cumple con lo necesario, sin embargo, no superan las expectativas de sus clientes, ello debido a la ausencia de buenas prácticas en gestión por procesos.

Se desarrolló un nuevo modelo de gestión por procesos analizando al hotel interna y externamente, se detallaron las actividades del proceso de reserva, su caracterización y ficha de indicador para el proceso en evaluación.

Concluyendo que la empresa se encuentra fuerte internamente con 2.64 en la matriz EFI y en la matriz EFE con 2.67, lo que significa un entorno favorable, por ello se establecieron 12 estrategias en la matriz DAFO. Se reestructuró el diagrama as is generando un proceso de reserva que contenga actividades digitales, se estimaron los costos de implementación con un total de S/9'204 y

Digital Object Identifier (DOI):

http://dx.doi.org/10.18687/LEIRD2021.1.1.9

ISSN: 2414-6390 ISBN: 978-958-52071-9-6 se elaboró la matriz de impacto social y ambiental con la finalidad de lograr una mayor sostenibilidad y competitividad en la organización.

Palabras clave - Gestión por procesos, competitividad, proceso de reserva, sostenibilidad, Covid-19.

\section{INTRODUCCIÓN}

El sector hotelero es una de las industrias del turismo más reconocidas y destacadas, debido a los aspectos culturales, organizacionales e innovadores, los cuales influyen en una adecuada gestión de la empresa generando ventajas competitivas para los hoteles [1]. Del mismo modo, este sector en desarrollo, caracterizado por pequeños negocios, generalmente familiares, deben establecer procedimientos competitivos para buscar la mejora de la organización en cuanto a sus servicios y la satisfacción de los clientes [2].

Los modelos de gestión responden a determinadas necesidades específicas de una organización, instituciones o empresas, que tienen como finalidad optimizar los recursos de los procesos de satisfacción de las necesidades de los clientes, teniendo en cuenta, el diseño y la estructuración de los mismos, los cuales tendrán variaciones para la mejora continua [3]. Es importante recalcar que se debe considerar que para la aplicación de la "Mejora de Procesos" se tiene que cumplir con las premisas siguientes: existencia de compromiso por parte de la alta dirección (responsables de la gerencia de la organización) sustentado en un trabajo en equipo donde se involucre a las personas con capacidad de aportar ideas, ejecutar las actividades y tomar las decisiones correctas [4].

Las herramientas empresariales para alcanzar las posiciones de alto nivel de competitividad son variadas; además del análisis de la cadena de valor se debe tomar en cuenta a las propuestas de análisis de procesos, la reingeniería de los procesos, entre otros y tomando como referencia a la gestión por procesos de los últimos años por la madurez que los procesos administrativos puedan tener y para proporcionar un modelo de gestión adecuado [5].

Para su gestión, parte desde la identificación de los procesos, definición de la relación entre ellos a través del mapa de procesos, descripción de las actividades través de los diagramas, apoyando la información con la ficha del proceso donde entran puntos de gran nivel de importancia, entre otros 
temas; se establecen indicadores que permite medir la capacidad, eficiencia y efectividad de los procesos. La información de los indicadores permitirá hacer un análisis de la operación, detectar oportunidades de mejora y tomar decisiones [5].

Por otro lado, la pandemia del COVID-19 en los últimos tiempos representa la prueba definitiva para numerosos empresarios, líderes y empleados que operan en su mayoría. Entre otros impactos, el contagio de virus ha afectado notablemente a la economía mundial incluidas las industrias de viajes, turismo y hotelería. Asimismo, la naturaleza de la COVID-19 ha tenido efectos paralizantes, con diversas restricciones en las empresas, lo que ha tenido como resultado impactos de gran medida en hoteles, bares, restaurantes y otros negocios de hostelería, en general desafíos insuperables en algunas empresas [6].

Cabe mencionar que la crisis actual del COVID-19 ha causado una caída de la actividad turística en todo el mundo, incluyendo a América Latina y el Caribe, debido a que muchos países cerraron sus fronteras desde marzo del año pasado por el contagio excesivo del virus, lo que originó una disminución del $95 \%$ en abril de 2020 y se estancación durante varios meses. Además, se prevé que la recuperación posterior tardará unos nueve meses, antes de llegar a mesetas del 50\%, el $60 \%$ y el $70 \%$ en el mundo. [7] En el Perú el COVID-19 ocasionó una caída en hoteles del $-90.6 \%$, en restaurants $-44.8 \%$ y en transportes aéreos $-41.2 \%$, lo que significa que fue el sector turismo el más golpeado por la crisis mundial sanitaria en territorio peruano [8].

Por último, Hotel Le Mans, es una empresa hotelera con años de trayectoria en la Ciudad de Trujillo - Perú, que ofrece servicio de hospedaje de 3 estrellas, brindando a sus clientes un trato acorde con los principios y valores de la organización. Además, ofrece a sus colaboradores la confianza lo cual refleja su convicción en el servicio, en el trabajo en equipo, y el compromiso para la empresa, tanto para su crecimiento profesional y personal. Por otro lado, la organización pretende desarrollarse y consolidarse como líder a nivel local, consiguiendo así ser la primera opción en los turistas u organizaciones para hospedarse encontrando comodidad y elegancia. Sin embargo, es de suma importancia mencionar que no se utilizan los recursos en su totalidad, ni se aprovechan las oportunidades de la industria y el trabajo dentro del hotel se desarrolla de manera cotidiana en base a los conocimientos de los hermanos responsables de la organización, quienes trabajaron en años anteriores en hoteles del País Japonés como colaboradores, pero sin un pensamiento estratégico para sus procesos. Concluyendo así que, si la empresa sigue trabajando de esta forma sin innovación, y sin un apropiado registro de sus reservas podría ser desplazada del mercado por empresas que están haciendo un adecuado uso de gestión por procesos para su competitividad y permanencia en el mercado. Obteniendo así el problema de investigación: ¿De qué manera la gestión de proceso determina la competitividad de la empresa hotelera Le Mans, frente al COVID-19 - Trujillo, 2021?

El objetivo general fue diseñar un modelo de gestión por procesos para la competitividad de la empresa hotelera Le Mans, frente al COVID-19 - Trujillo, 2021, y como objetivos específicos:

- Analizar la situación actual de la empresa hotelera, Le Mans.

- Describir el modelo de gestión por procesos para la empresa hotelera, Le Mans.

- Estimar los costos del modelo de gestión por procesos a la empresa hotelera.

- Determinar el impacto social y ambiental para el modelo propuesto.

\section{MetodologíA}

Para el desarrollo de la investigación se determinó la siguiente secuencia: El diseño de la investigación, la revisión del estado del arte, y el modelo de gestión por procesos.

\section{A. Diseño de la investigación}

A.1 Tipo de investigación

Según el propósito es aplicada, según el diseño de investigación es prospectiva, según el grado es descriptiva y según el tipo de datos empleados es mixta.

\section{A.2 Población y muestra}

La población estuvo constituida por todos los procesos que la empresa realiza, los 5 empleados que laboran y por todos los clientes del primer semestre del 2021. La muestra estuvo constituida por el proceso de reserva, además por 2 empleados del hotel y por 27 clientes los cuales fueron obtenidos mediante el muestreo por conveniencia, es una técnica de muestreo no probabilístico que permite seleccionar la muestra de acuerdo con la facilidad de acceso, la disponibilidad, en un intervalo de tiempo dado u otras especificaciones en particular [9].

\section{A.3 Procedimiento}

Se realizó una entrevista al Gerente General para obtener información relativa a la situación actual de la empresa y su desempeño en base a la gestión comercial realizada, mediante una guía de entrevista con preguntas pre-elaboradas. Se recolectó información necesaria para el análisis de la satisfacción del cliente tanto interno como externo, haciendo uso de dos cuestionarios estructurados validados por juicio de expertos y verificada su confiabilidad mediante el alfa de Cronbach con un valor de 0.736. Por último, mediante la revisión, información bibliográfica, análisis de datos relacionados con las variables de estudio, entre otros; se diseñó el modelo de gestión por procesos para el Hotel Le Mans.

\section{B. Estado del arte \\ B. 1 Procesos}

Según [10] define que un proceso es un conjunto de actividades de trabajo interrelacionadas, que se caracterizan por requerir ciertos insumos y actividades específicas que buscan agregar valor, para obtener ciertos resultados (outputs). Además, los procesos se clasifican en: procesos estratégicos, claves y de apoyo. Los estratégicos, permiten definir y desplegar cada estrategia y objetivo en la empresa; los procesos claves añaden valor al cliente o inciden de forma directa en su satisfacción y 
los de apoyo son necesarios para controlar y mejorar la gestión de los procesos.

\section{B.2 Gestión por procesos}

Actualmente, se define un nuevo concepto de estructura organizativa, que tiene en consideración la concepción de una red de procesos interconectados entre sí, a la cual se puede aplicar un modelo de gestión denominado Gestión basada en Procesos (GbP) [10].

[10] Empresas líderes han aplicado el cambio en su organización mediante la individualización de sus procesos y eligiendo los más relevantes para posteriormente analizarlos, mejorarlo hasta utilizar este enfoque para transformar sus empresas. Logrando así que la organización se oriente hacia la calidad y optimización de los procesos, esto implica que la empresa continuamente desarrolle de manera eficiente y eficaz la interrelación de sus procesos clave obteniendo resultados positivos que influyen en el cliente final [11].

Con la gestión por procesos se puede obtener amplios beneficios [12], por ejemplo:

- Aplicar fórmulas de costeo a los procesos, a nivel de las actividades para saber realmente cuánto cuestan nuestros productos o servicios.

- Aplicar métodos de mejora continua, que aseguren la calidad que nos permita aumentar la eficiencia y la eficacia.

- Rediseñar un proceso para conseguir rendimientos mayores.

- Innovar a diferentes niveles: proceso, actividad y tarea.

- Realizar de forma verídica el control de gestión, porque parte del cambio en los procesos consiste en obtener información relevante, tal como incorporar indicadores en tiempo real y adecuadamente comparados en el tiempo.

\section{B.3 Competitividad}

[13] El término competitividad ha evolucionado en gran magnitud $y$ se ha incorporado distintos elementos como cambios productivos, tecnológicos y organizacionales. A raíz de estos cambios, se empieza a analizar la competitividad en distintos ámbitos: internacional, nacional, sectorial y empresarial. Para un enfoque más específico, se debe hacer análisis a cuatro niveles: nivel meta: capacidad nacional de conducción; nivel macro: condiciones macroeconómicas estables; nivel meso: formación de estructuras y políticas selectivas y nivel micro: eficiencia, calidad, rapidez y flexibilidad, pese a ser importante lo anterior, es en la empresa, en donde la competitividad emerge.

[14] Desde la visión microeconómica, se dice que una empresa es competitiva cuando posee aquellos factores que le permiten disponer de ciertas ventajas para lograr un desempeño superior al de sus competidores.

Los autores también señalan que la ventaja competitiva se crea combinando la dotación de recursos que la empresa dispone con una adecuada estrategia en la cual se involucren dichos recursos, aptitudes de todo el personal, así como oportunidades que ofrece el medio ambiente, todo ello con el fin de asegurar el crecimiento y rentabilidad de la empresa.
Según el art. 2 de la Ley 28015, se define a la MYPE (Micro y Pequeña empresa) como la unidad económica constituida por una persona jurídica o natural, que tiene como objetivo desarrollar distintas actividades como de: extracción, transformación, producción, comercialización de bienes o la prestación de servicios. Además, según el art. 3 de la Ley 28015, las MYPE deben contener las siguientes características concurrentes:

- Microempresa: de uno (1) hasta diez (10) trabajadores incluyendo ventas anuales hasta el monto máximo de 150 Unidades Impositivas Tributarias (UIT).

- Pequeña Empresa: de uno (1) hasta cien (100) trabajadores incluyendo ventas anuales hasta el monto máximo de 1700 Unidades Impositivas Tributarias (UIT).

Señalando el estudio de [15] las MYPES son el motor de la economía en Perú. A nivel nacional. las micro y pequeñas empresas brindan empleo al $80 \%$ de la población que se encuentra económicamente activa y generan aproximadamente el $40 \%$ del Producto Bruto Interno (PBI).

\section{B.5 Definiciones conceptuales}

Se presenta los principales conceptos utilizados en la investigación tomando como referencia [16]

FODA: Herramienta conceptual para un análisis sistemático que permite determinar estrategias dentro de una empresa para utilizar las fortalezas, minimizar debilidades, aprovechar oportunidades y evitar amenazas.

Las Cinco fuerzas de Porter: Herramienta microeconómica para analizar el atractivo de un sector industrial la cual de una forma sencilla y clara ayuda a comprender la dinámica competitiva del sector a partir de la interrelación de sus cinco componentes las cuales permiten evaluar la competencia y desarrollar estrategias.

MEFE: Matriz que evalúa los factores externos de la empresa (amenazas y debilidades) y determinan que tan fuerte es la posición externa de la empresa en el mercado.

MEFI: Matriz que evalúa los factores internos (debilidades y fortalezas) y determinan que tan fuerte es la posición interna de la empresa.

DAFO: Matriz que establece estrategias de acuerdo con la relación de las debilidad, amenazas, fortalezas y oportunidades. PESTEL: Matriz para evaluar factores: Políticos, económicos, sociales, tecnológicos, ecológicos, y legales.

\section{Diseño del modelo de gestión por procesos}

El procedimiento del diseño empezó con la obtención de información por parte de la Gerencia, contemplando el eje estratégico (misión, visión, etc.), el proceso de reserva del Hotel Le Mans, de los cuales se realizó el análisis de datos mediante las técnicas e instrumentos.

Tras ello, se realizó el mapa de procesos de la empresa en donde se seleccionó a los procesos con los cuales se hizo las evaluaciones a clientes internos y externos. Además, para la reestructuración de los procesos se plasmaron en diagramas de Bizagi As Is y posteriormente los To Be, junto con su ficha de indicadores y caracterización.

\section{B.4 MYPE}


Finalmente, se obtuvo el diseño completo de la gestión por procesos, la estimación de costos y el impacto social y Ambiental de los procesos.

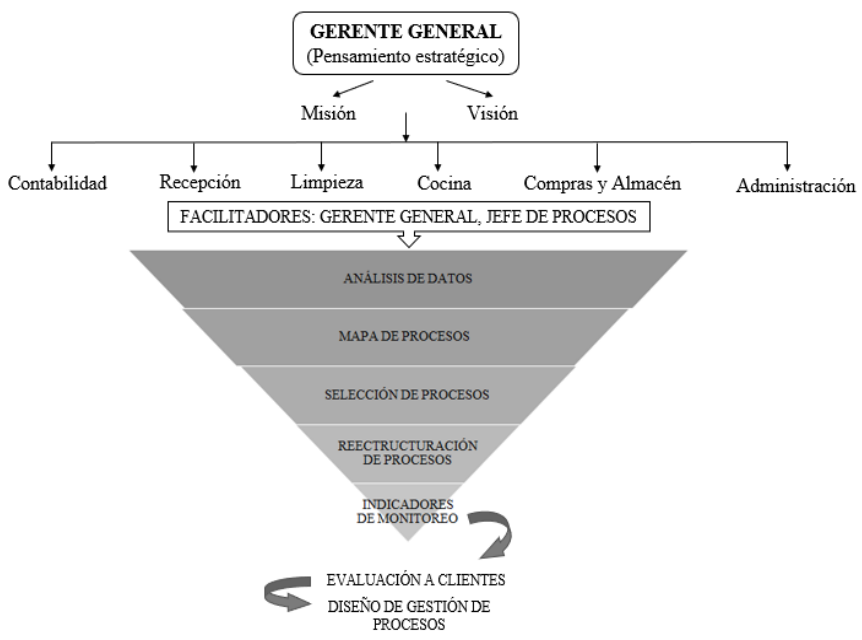

Fig. 1 Diseño del modelo de gestion de procesos Elaroración propia

\section{RESULTADOS}

A continuación, se presentan los principales resultados de acuerdo a cada etapa del diseño de gestión por procesos.

\section{A. ETAPA I: Análisis de la Situación Actual}

\section{A.1 Análisis Interno}

De las entrevistas realizadas se determinó:

Misión: Somos una empresa privada, líder en la prestación de servicios hoteleros, que ofrece una atención personalizada y amable con calidad. Centrándonos en la honradez y permanente limpieza del hotel; desarrollando servicios que garantizan la satisfacción y las expectativas del cliente. Realizando un mejoramiento continuo, que permite ampliar y mantener el mercado brindando total seguridad a empleados, clientes, proveedores, socios y comunidad en general.

Visión: Ser de las empresas más reconocidas dentro del rubro hotelero en la ciudad de Trujillo, fomentando el desarrollo empresarial y turístico de la ciudad, brindando el mejor servicio personalizado y comodidad que se puede ofrecer a través de la mejora continua.

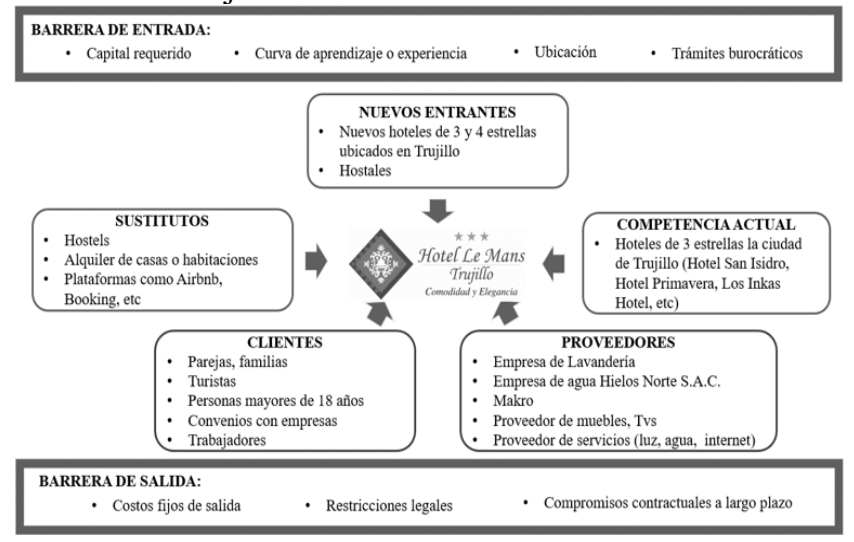

Fig. 2 Las 5 Fuerzas de Porter del Hotel Le Mans

Elaboración Propia
La Fig. 2 representa a las 5 Fuerzas de Porter para analizar el entorno con el cual la empresa compite, conociéndose a los nuevos competidores, a los sustitutos, a la competencia actual del Hotel, la segmentación de sus clientes y clasificación de proveedores.

TABLA I

MATRIZ DE EVALUACIÓN DE FACTORES INTERNOS (MEFI)

\begin{tabular}{|c|c|c|c|}
\hline \multicolumn{4}{|c|}{ MATRIZ EFI } \\
\hline \multicolumn{4}{|c|}{ FORTALEZAS } \\
\hline FACTOR CRÍTICO DE EXITO & VALOR & CALIF. & $\begin{array}{l}\text { CALIF. } \\
\text { PONDERADA }\end{array}$ \\
\hline 1. Ubicación estratégica & 0.09 & 4 & 0.36 \\
\hline 2. Entorno laboral favorable & 0.13 & 3 & 0.39 \\
\hline 3. Buena atención al cliente & 0.11 & 4 & 0.44 \\
\hline $\begin{array}{l}\text { 4. Aplicación de promociones } \\
\text { 5. Buena distribución de la }\end{array}$ & 0.09 & 3 & 0.27 \\
\hline infraestructura & 0.11 & 3 & 0.33 \\
\hline SUBTOTAL & 0.53 & & 1.79 \\
\hline \multicolumn{4}{|c|}{ DEBILIDADES } \\
\hline FACTOR CRÍTICO DE EXITO & VALOR & CALIF. & $\begin{array}{c}\text { CALIF. } \\
\text { PONDERADA }\end{array}$ \\
\hline 1. Baja presencia en redes sociales & 0.10 & 1 & 0.10 \\
\hline 2. No realiza alianzas estratégicas & 0.11 & 2 & 0.22 \\
\hline 3. Tarifa alta & 0.09 & 2 & 0.18 \\
\hline 4. Inexistencia de base de datos & 0.09 & 3 & 0.27 \\
\hline 5. Desorganización de funciones & 0.08 & 1 & 0.08 \\
\hline SUBTOTAL & 0.47 & & 0.85 \\
\hline TOTAL & 1.00 & & 2.64 \\
\hline
\end{tabular}

Elaboración Propia

La Tabla I muestra a la matriz EFI con un valor: $0 \%=\sin$ importancia, $100 \%=$ muy importante y a las condiciones: $1=$ mayor debilidad, $2=$ menor debilidad, $3=$ menor fortaleza $\mathrm{y}$ $4=$ mayor fortaleza. Se observa que el subtotal de fortalezas es 1.79 , siendo mayor a las debilidades 0.85 . El resultado total es 2.64, superando el puntaje promedio de 2.50 e indicando un escenario favorable para el crecimiento de la empresa.

\section{A.2 Análisis Externo}

La matriz EFE califica al valor: $0 \%=\sin$ importancia, $100 \%=$ muy importante $\mathrm{y}$ a las condiciones: $1=$ mayor amenaza, 2=menor amenaza, 3=menor oportunidad, 4=mayor oportunidad. En la Tabla II se observa que la ponderación de las oportunidades es 1.75, estando por encima de las amenazas 0.92 . Su resultado total es 2.67 , encontrándose por encima del promedio de 2.50 , lo cual significa que la empresa está haciendo uso efectivo de las oportunidades externas para neutralizar las amenazas, aun teniendo en cuenta siempre que se pueden mejorar el uso de las oportunidades. 
TABLA II

MATRIZ De Evaluación DE FACTORES EXTERnOS (MEFE)

\begin{tabular}{|c|c|c|c|}
\hline \multicolumn{4}{|c|}{ MATRIZ EFE } \\
\hline \multicolumn{4}{|c|}{ OPORTUNIDADES } \\
\hline FACTOR CRÍTICO DE EXITO & VALOR & CALIF. & $\begin{array}{c}\text { CALIF. } \\
\text { PONDERADA }\end{array}$ \\
\hline 1. Problemas económicos de la competencia & 0.11 & 3 & 0.33 \\
\hline 2. Incremento de publicidad por internet & 0.12 & 4 & 0.48 \\
\hline 3. Capacitaciones de empresas recurrentes. & 0.10 & 4 & 0.40 \\
\hline 4. Pase libre a los museos & 0.09 & 4 & 0.36 \\
\hline 5. Financiamiento externo & 0.09 & 2 & 0.18 \\
\hline SUBTOTAL & 0.51 & & 1.75 \\
\hline \multicolumn{4}{|c|}{ AMENAZAS } \\
\hline FACTOR CRÍTICO DE EXITO & VALOR & CALIF. & $\begin{array}{c}\text { CALIF. } \\
\text { PONDERADA }\end{array}$ \\
\hline 1. Crisis económica & 0.11 & 3 & 0.33 \\
\hline 2. Estado de emergencia & 0.11 & 2 & 0.22 \\
\hline 3. Aumento de precios de insumos & 0.10 & 2 & 0.20 \\
\hline 4. Reducción de tarifas de los competidores & 0.09 & 1 & 0.09 \\
\hline 5. Falta de seguridad & 0.08 & 1 & 0.08 \\
\hline SUBTOTAL & 0.49 & & 0.92 \\
\hline TOTAL & 1.00 & & 2.67 \\
\hline
\end{tabular}

Elaboración Propia

TABLA III

MATRIZ DAFO (DEBILIDADES, AMENAZAS, FORTALEZAS Y OPORTUNIDADES)

\begin{tabular}{|c|c|c|}
\hline \multirow{6}{*}{ MATRIZ DAFO } & FORTALEZAS & DEBILIDADES \\
\hline & F1. Ubicación estratégica & D1. Baja presencia en redes sociales \\
\hline & F2. Entorno laboral favorable & D2. No realiza alianzas estratégicas \\
\hline & F3. Buena atención al cliente & D3. Tarifa alta \\
\hline & F4. Aplicación de promociones & D4. Inexistencia de base de datos \\
\hline & $\begin{array}{l}\text { F5. Buena distribución de la } \\
\text { infraestructura }\end{array}$ & D5. Desorganización de funciones \\
\hline OPORTUNIDADES & ESTRATEGIAS FO & ESTRATEGIAS DO \\
\hline $\begin{array}{l}\text { O1. Problemas económicos de } \\
\text { la competencia }\end{array}$ & \multirow{5}{*}{$\begin{array}{c}\text { O2-F2-F4. Realizar } \\
\text { promociones en festividades } \\
\text { importantes durante el año. } \\
\text { O1-O2-O4-F2. Crear contenido } \\
\text { para las redes sociales. } \\
\text { O1-O3-04-F2-F4-F1. Ofrecer } \\
\text { descuento por el número de } \\
\text { visitas }\end{array}$} & \multirow{5}{*}{$\begin{array}{c}\text { O1-D4 Brindar desayuno } \\
\text { continental gratis } \\
\text { O5-04-D1-D2 Realizar alianzas } \\
\text { estratégicas con empresas de viajes } \\
\text { O5-03-O1-D1-D2 Realizar un plan } \\
\text { de marketing }\end{array}$} \\
\hline $\begin{array}{l}\text { O2. Incremento de publicidad } \\
\text { por internet }\end{array}$ & & \\
\hline $\begin{array}{l}\text { O3. Capacitaciones de } \\
\text { empresas recurrentes. }\end{array}$ & & \\
\hline 04. Pase libre a los museos & & \\
\hline O5. Financiamiento externo & & \\
\hline AMENAZAS & ESTRATEGLAS FA & ESTRATEGIAS DA \\
\hline A1. Crisis económica & \multirow{5}{*}{$\begin{array}{l}\text { A1-A2-A4-F2-F4 Actualizar } \\
\text { tarifas de precios de las } \\
\text { habitaciones } \\
\text { A1-A2-A3-F2 Búsqueda de } \\
\text { nuevos proveedores } \\
\text { A1-A2-A5-F2-F1 Reubicar las } \\
\text { cámaras de seguridad para } \\
\text { mayor vigilancia }\end{array}$} & \multirow{5}{*}{$\begin{array}{l}\text { A1-A2-D4 Insertar un sistema de } \\
\text { almacenamiento de datos } \\
\text { A1-A2-D3 Actualizar tarifas de } \\
\text { precios de habitaciones } \\
\text { A1-A2-D5 Delimitar las funciones } \\
\text { de los empleados }\end{array}$} \\
\hline A2. Estado de emergencia & & \\
\hline $\begin{array}{l}\text { A3. Aumento de precios de } \\
\text { insumos }\end{array}$ & & \\
\hline $\begin{array}{l}\text { A4. Reducción de tarifas de } \\
\text { los competidores }\end{array}$ & & \\
\hline A5. Falta de seguridad & & \\
\hline
\end{tabular}

Elaboración Propia

En la Tabla III se muestra a la matriz DAFO (debilidades, amenazas, fortalezas y oportunidades), en la que se establecieron 12 estrategias para aprovechar los recursos de la empresa y cumplir con sus objetivos.

\section{A.2 Análisis de Encuestas}

Se realizó el análisis de datos de las encuestas realizadas a 27 clientes del Hotel Le Mans para conocer su opinión y hacer un modelo de gestión por procesos que se adecue tanto a la empresa como a la satisfacción de sus clientes.

\section{Rango de edades de los clientes}

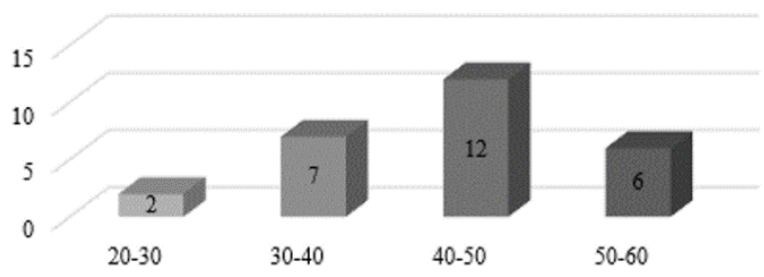

Fig. 3 Rango de edades de Clientes Encuestados Elaboración Propia

En la Fig. 3 se determinó el rango promedio de las edades, en la cual se obtuvo que 2 clientes pertenecen al rango (20-30 años), 7 clientes son de (30-40 años), 12 clientes encuestados constituyen a rango de (40-50 años) y 6 de los clientes encuestados son del rango de (50-60 años), haciéndose notar que el rango de edad destacado es el de 40-50 años de edad.

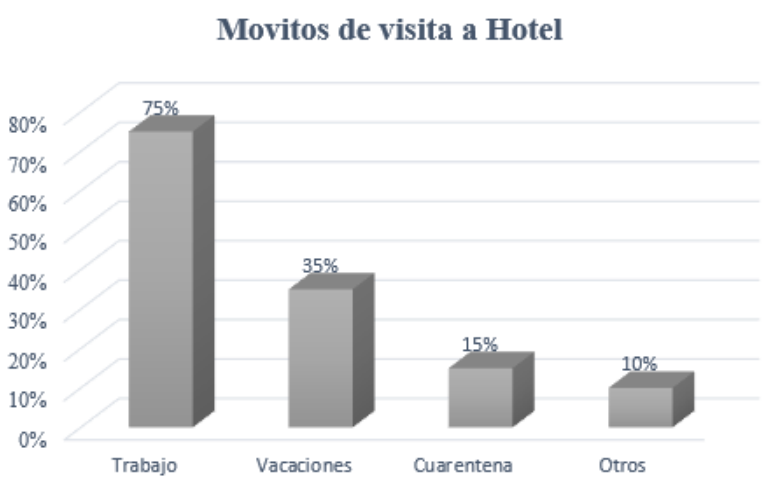

Fig. 4 Motivos de visita al Hotel Le Mans Elaboración Propia

En la Fig. 4 se denota que el $75 \%$ de los encuestados se alojó en el hotel por motivos de trabajo, el motivo que le siguió es el de vacaciones con respuesta del 35\% de encuestados, además, por motivos de cuarentena obligatoria, el porcentaje fue de $15 \%$ y por otros motivos (por horas, salud, etc.) fue el $10 \%$ del total de encuestados.

\section{Análisis de Atención a Clientes Femeninos}

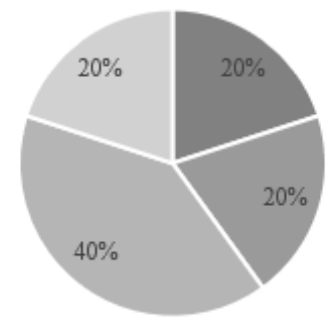

- Siempre - Frecuentemente "Regularmente " Muy Poco

Fig. 5 Análisis de Atención al Cliente del Género Femenino Elaboración Propia 
La Fig. 5 muestra los gráficos de la plataforma Qlik Sense para conocer la opinión de las clientes femeninas según su rango de edad, en la cual se conoció que en el rango 20-30 años opina que la atención es rápida regularmente y muy poco, además en el rango de 30-40 años opina que la atención siempre es rápida y la del rango de 40-50 años es frecuente y regularmente, en donde se entendió que habían diferencias de acuerdo al horario de entrada de los clientes ya que el turno en la recepción cambia.

\section{B. ETAPA 2: Diseño del Modelo de Gestión por Procesos \\ B.1 Mapa de Procesos To Be}

La Fig. 6 muestra al mapa de procesos To Be del Hotel Le Mans con los 3 ejes principales, en el eje estratégico se encuentra: gestión administrativa, publicidad en redes y planeación estratégica, en el eje operativo: el proceso de atención al cliente, gestión de cocina y facturación y en el eje de soporte: proceso de recursos humanos, de seguridad y protección, desinfección y de gestión de almacén, ello se recomienda para mantener una mejor gestión por procesos.

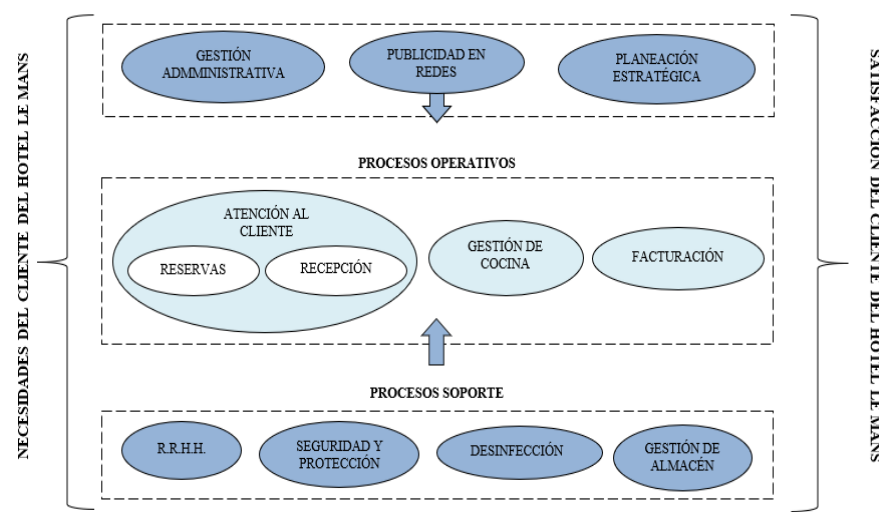

Fig. 6 Mapa de Procesos To Be Elaboración Propia

\section{B.2 Diagramas de Flujo de Reserva As Is}

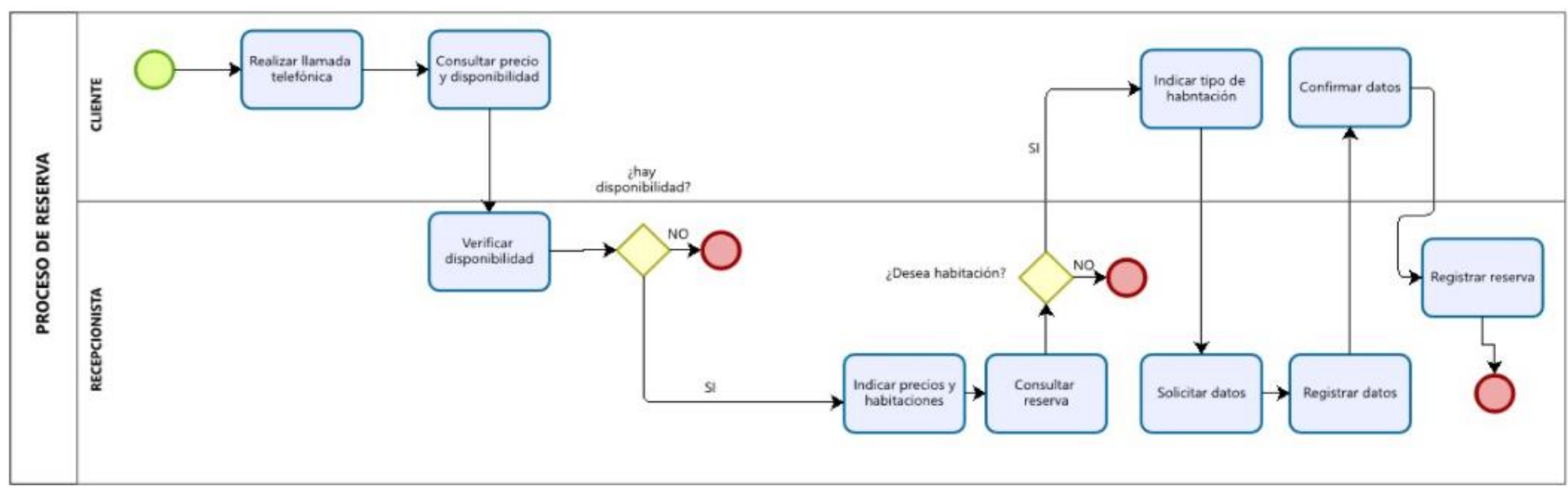

Fig. 7 Diagrama de Flujo de Reserva As Is Elaboración Propia

\section{B.3 Diagramas de Flujo de Reserva To Be}

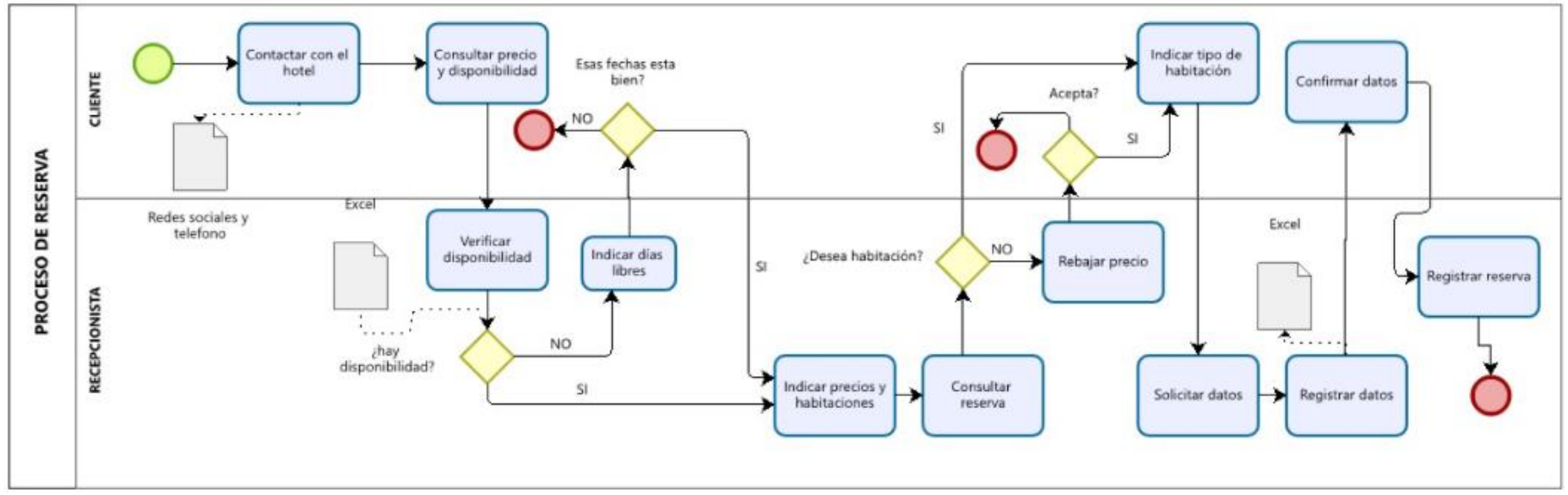

Fig. 8 Diagrama de Flujo de Reserva To Be

Elaboración Propia 
En la Fig. 7 se observa el diagrama de flujo As Is de reserva de la empresa Hotel Le Mans en la cual participa el recepcionista de la empresa y un cliente que desee reservar una o más habitaciones en la empresa, este proceso se realiza vía telefónica para conocer la disponibilidad de habitaciones, cotizar precios y fechas, para luego poder realizar el registro de la reserva. Además, es sabido que suelen registrar las reservas de forma manual, con lo cual han tenido inconvenientes o problemas por distintos motivos (correcciones, registro inadecuado, hojas rotas o mojadas, entre otros).

La Fig. 8 muestra al proceso de reserve to be reformado, donde se añade la utilización de las redes sociales para el contacto con el hotel, haciendo énfasis en WhatsApp Business, para que la empresa ofrezca su catálogo de servicios en el mismo y se vea más formal cuando la contacten. Asimismo, se busca hacer uso de Microsoft office para el registro de datos en el sistema, ya que suelen escribir en un cuaderno aparte, con el cual han tenido inconvenientes o problemas de registro, por ello para trabajar de manera eficiente se optó por dichas mejoras, además al tener la información siempre actualizada en la base de datos del Excel, permitiría responder de manera rápida y eficiente las dudas o consultas que el cliente solicite.

\section{B.4 Caracterización del Proceso}

TABLA IV

CARACTERIZACIÓN DEL PROCESo DE RSERVA

\begin{tabular}{|c|c|c|c|c|c|c|}
\hline \multicolumn{7}{|c|}{ FICHA TÉCNICA DEL PROCESO DE RESERVA } \\
\hline Nombre & \multicolumn{3}{|c|}{ RESERVA } & Responsable & \multicolumn{2}{|c|}{ RECEPCIONISTA } \\
\hline Objetivo & \multicolumn{3}{|c|}{ Realizar de manera eficiente la reserva del cliente, brindando la información correcta. } & Requisitos & \multicolumn{2}{|c|}{$\begin{array}{l}\text { Informe de disponibilidad de } \\
\text { habitaciones }\end{array}$} \\
\hline Alcance & \multicolumn{3}{|c|}{ Contactar con el hotel/ registrar reserva } & Clasificación & \multicolumn{2}{|c|}{ Proceso Operativo } \\
\hline \multicolumn{7}{|c|}{ DESCRIPCIÓN DEL PROCESO } \\
\hline Proveedores & Entradas & Actividades & \multicolumn{3}{|c|}{ Salidas } & $\begin{array}{c}\text { Destinatario de los } \\
\text { bienes y servicios }\end{array}$ \\
\hline Cliente & Información de contacto & Contactar con el hotel & \multicolumn{3}{|c|}{ Número de contacto del hotel o redes } & Cliente \\
\hline Cliente & Número de contacto del hotel o redes & Consultar precio y disponibilidad & \multicolumn{3}{|c|}{$\begin{array}{l}\text { Solicitud de consulta de precio y } \\
\text { disponibilidad }\end{array}$} & Recepcionista \\
\hline Recepcionista & $\begin{array}{l}\text { Solicitud de consulta de precio y } \\
\text { disponibilidad }\end{array}$ & Verificar disponibilidad & \multicolumn{3}{|c|}{ Lista de precios y disponibilidad } & Recepcionista \\
\hline Recepcionista & Lista de precios y disponibilidad & Indicar dias libres & \multicolumn{3}{|c|}{ Ficha de fechas libres } & Cliente \\
\hline Recepcionista & Lista de precios y disponibilidad & Indicar precios y habitaciones & \multicolumn{3}{|c|}{$\begin{array}{l}\text { Ficha de precios y habitaciones } \\
\text { disponibles }\end{array}$} & Cliente \\
\hline Recepcionista & Conformidad de reserva & Consultar reserva & \multicolumn{3}{|c|}{ Ficha de posible reserva } & Cliente \\
\hline Recepcionista & Lista de promociones o descuentos & Rebajar precio & \multicolumn{3}{|c|}{ Ficha de posible reserva } & Cliente \\
\hline Cliente & Requerimientos de habitación & Indicar tipo habitación & \multicolumn{3}{|c|}{ Especificación de habitación } & Recepcionista \\
\hline Recepcionista & Solicitud de datos & Solicitar datos & \multicolumn{3}{|c|}{ Toma de datos } & Cliente \\
\hline Recepcionista & Toma de datos & Registrar datos & \multicolumn{3}{|c|}{ Ficha de reserva } & Cliente \\
\hline Cliente & Ficha de reserva verificada & Confirmar datos & \multicolumn{3}{|c|}{ Ficha de reserva registrada } & Recepcionista \\
\hline Recepcionista & Ficha de reserva registrada & Registrar reserva & \multicolumn{3}{|c|}{ Reserva correctamente registrada } & Recepcionista \\
\hline \multicolumn{7}{|c|}{ IDENTIFICACIÓN DE RECURSOS CRÍTICOS PARA LA EJECUCIÓN DEL PROCESO } \\
\hline & Responsables & \begin{tabular}{|l|} 
Parámetros de Control/ \\
Medición/Seguimiento \\
\end{tabular} & \multicolumn{4}{|c|}{ Documento / Registros } \\
\hline & Recepcionista & $\begin{array}{l}\text { (Reservas registradas en linea } \\
\text { Total de reservas) } * 100 \%\end{array}$ & \multicolumn{4}{|c|}{$\begin{array}{l}\text { Registro de reservas, ficha de reserva, disponibilidad de } \\
\text { habitaciones y actualización de precios. }\end{array}$} \\
\hline \multicolumn{7}{|c|}{ EVIDENCIAS E INDICADORES DEL PROCESO } \\
\hline \multicolumn{4}{|c|}{ Proceso de Soporte } & \multicolumn{3}{|c|}{ Requisitos para cumplir } \\
\hline \multicolumn{4}{|c|}{ Proceso de Recepción } & \multicolumn{3}{|c|}{ Registro correcto de los datos de la reserva } \\
\hline
\end{tabular}

Elaboración Propia 
En la Tabla IV se observa a la ficha de caracterización del proceso de reserva, la cual contiene la descripción de cada actividad del proceso con sus respectivos proveedores, entradas, salidas y destinatarios, además de los parámetros a cumplir, los indicadores, sus requisitos $\mathrm{y}$ registros $\mathrm{o}$ documentos necesarios.

\section{ETAPA 3: Estimación de Costos de Implementación}

En la Tabla V se denota el resumen de los costos total estimados para la implementación del modelo, el cual contiene los costos del equipo de proyecto por 3 meses con un total de $\mathrm{S} / 6$ ' 410 y los costos de materiales, equipos y servicios con una suma de S/2'794. Su costo total es de S/9'204.

TABLA V

RESUMEN DE ESTIMACIÓN DE COSTOS

\begin{tabular}{lc}
\hline \multicolumn{2}{c}{ RESUMEN DE COSTOS } \\
\hline \multicolumn{1}{c}{ COSTO } & SUBTOTAL \\
Equipo de proyecto & $\mathrm{S} / 6^{\prime} 410.00$ \\
Materiales, equipos y servicios & $\mathrm{S} / 2^{\prime} 794.00$ \\
TOTAL & $\mathrm{S} / \mathbf{9}^{\prime} \mathbf{2 0 4 . 0 0}$ \\
\hline
\end{tabular}

Elaboración Propia

\section{ETAPA 4: Impacto Social y Ambiental del Modelo}

Se determinó el impacto ambiental y social que puede enfrentar el proceso de reserva en el diseño del modelo de gestión:

Impacto Social: Se busca contar a un personal idóneo para el desarrollo eficaz de los procesos involucrados, donde se les brindaría un seguro de salud, una subvención económica y conocimientos que incrementen su capacidad cognitiva de manera sobresaliente. Asimismo, se insertará los principios básicos para conseguir un ambiente laboral ordenado y armonioso, respetando su cultura, raza y religión.

Impacto Ambiental: Mediante el proceso de reserva en línea de habitaciones se estará reduciendo la contaminación ambiental, evitando el traslado en carro lo que reduciría el dióxido de carbono que emiten las movilidades de la ciudad, por otro lado, se evitará el uso de papel y se generará todo el registro de huéspedes y reserva de manera digital.

\section{DISCUSIÓN}

Tomando como base los resultados de las encuestas de los clientes, se pudo verificar que el motivo mayor de asistencia al hotel es por trabajo con un total de 15 clientes, 7 por vacaciones, 3 por cuarentena obligatoria y 2 por otras razones, y que son las damas quienes no están conforme con la atención al cliente en su mayoría con un porcentaje del $40 \%$,el rango de edades de los clientes resalta entre 40 a 50 años y 30 a 40 años con un total de 12 y 7 clientes respectivamente, cabe recalcar que la cifra de encuestados no es el promedio histórico de la empresa, ya que antes de la pandemia la cifra mensual era de 80 a 90 clientes, sin embargo, esto se utilizó para establecer nuevas actividades dentro del proceso de reserva para generar competitividad en la

En el informe se realizaron encuestas a 27 clientes de la empresa hotelera, para las cuales se utilizó el análisis de coeficiente de Alfa de Cronbach para interpretar el grado de confiabilidad de los resultados obteniéndose una puntuación de 0.736, la información de las encuestas permitió analizar al cliente y qué es lo que se debe mejorar en los procesos para poder fidelizarlos. Además, en la tesis de [17] se menciona como parte de la metodología a una encuesta y cuestionario con la escala Likert a 384 consumidores, y su investigación tiene un resultado de 0.678 lo que le indica la confiabilidad de sus resultados.

Se muestra en la matriz EFI con un ponderado de 2.64, lo cual está por encima de los 2.5 , lo que significa que la empresa internamente se encuentra fuerte y comprueba con una puntuación en sus fortalezas de 1.79 versus el ponderado de las debilidades que es de 0.85 , lo cual beneficia la creación de estrategias en la matriz DAFO y el aprovechamiento de las oportunidades, y en la matriz EFE se obtuvo un ponderado de 2.67 con una puntuación de 1.75 en las oportunidades que se encuentra por encima de las amenazas de 0.92, lo que significa que el entorno donde se desarrolla es favorable y se pueden aprovechas las fortalezas de la organización, entonces en base a estos resultados se inserta procesos de forma digital, respondiendo así a los intereses de los clientes. Lo que confirma [18] cuando manifiesta que el éxito de toda organización depende cada vez más de que sus procesos comerciales estén alineados a sus objetivos y estrategias, y concluye que una herramienta de apoyo es la construcción adecuada de los procesos con un soporte electrónico o apoyado en la tecnología.

Además, es necesario recalcar que el aspecto tecnológico es de fundamental apoyo para la gestión por procesos, porque permite optimizar las actividades y tareas de los procesos, es por ello que, el proceso de reserva han sido estructurado con el uso de la tecnología, lo cual apoya a la búsqueda de la satisfacción del cliente y contribución con el medio ambiente, reduciendo el uso de recursos físicos, lo cual es confirmado por el artículo científico de [18] quienes afirman que el éxito competitivo de las empresas es influenciada de forma positiva por la innovación, tecnología y la calidad en su servicio o producto.

\section{CONCLUSIONES}

Se diseñó el modelo de gestión por procesos del hotel Le Mans como se denota en la Fig. 1, de manera detallada con la finalidad de incrementar los niveles de competitividad en la empresa frente al COVID-19, el cual inicia con el análisis de datos de las encuestas a los clientes y colaboradores así como la entrevista con el gerente, se plasmó el mapa de procesos, se seleccionaron los procesos claves de la organización, y se escogió al proceso de reserva además, se reestructuró las 
actividades del proceso seleccionado, donde se realizaron mejoras mediante la utilización de herramientas digitales, se planteó un indicador de monitoreo para el proceso en estudio, se estimaron los costos de implementación y se elaboró la matriz de impacto social y ambiental de los procesos para la sostenibilidad de la organización.

Se analizó la situación actual de la empresa hotelera mediante distintas matrices conociéndose que se encuentra fuerte internamente, debido a la ponderación de 2.64 en la matriz EFI, además en la matriz EFE el hotel se encuentra en un entorno favorable con un ponderado de 2.67 , lo que comprueba que la realización de las 12 estrategias planteadas como: agregar a la tarifa un plus de cortesía como el desayuno oriental, establecer las funciones para cada empleado, insertar uso de la tecnología en los procesos y el aprovechamiento de las oportunidades generaría un alto nivel de competitividad en su sector. Asimismo, se analizó a 27 clientes mediante encuestas donde se constató que las mujeres son más minuciosas en cuanto a la limpieza y atención del personal a cargo, así mismo se verificó que los clientes más concurridos al hotel son aquellos que van por trabajo, y lo que permitió las mejoras respecto a los procesos.

Se describió de manera organizada y clara el modelo de gestión de los procesos, se inició con la modificación del mapa de procesos y se realizó nuevo diagrama de flujo, modificando y agregando actividades que permitan mejorar el funcionamiento del proceso de reserva, se insertó el uso de reserva vía online y el registro digital de reservas en la $\mathrm{PC}$ mediante Excel, por lo cual se creó la caracterización de proceso especificando a detalle las actividades, recursos, y también se estableció un indicador para que permitan medir el desempeño del proceso y se detallaron los objetivos, alcance y responsables para que se pueda ejecutar de mejor forma.

Se estimó el costo implementación de la gestión por procesos, donde se conoció el costo del personal equivalente a $\mathrm{S} / 6$ '410 y los costos de los materiales, equipos, luz e internet que serían de S/2'794, todo ello suma S/9'204, como el total de los costos para la implementación del modelo de gestión por procesos para incrementar la competitividad de la organización.

Se determinó el impacto ambiental y social para los procesos operativos de la organización a través de una matriz, en la cual se establece un clima laboral adecuado, incrementación de las oportunidades labores, respetando los derechos de cada cliente interno y externo, brindándoles los beneficios correspondientes a la ley y otorgándoles la libertad de expresarse en cualquier situación, con el fin mejorar el impacto social, por otro lado, para reducir el impacto ambiental se planteó una reducción de papel, agua y reutilización de recursos plásticos en el Hotel Le Mans.

\section{AGRADECIMIENTO}

Queremos expresar nuestro agradecimiento al Gerente de la empresa Hotelera Le Mans y a nuestro Asesor por el apoyo brindado.

\section{REFERENCIAS}

[1] León, Padrón-Díaz, Estephany Alejandra, y Elva Esther. s. f. «Cultura organizacional e innovación en el sector hotelero: estado del conocimiento». 22.

[2] Cedeño, H.; Real-Pérez, L. (2020) «Competitividad y calidad en pequeños hoteles $\mathrm{o}$ en desarrollo. Caso hotel Amash». Recuperado de https://dialnet.unirioja.es/servlet/articulo?codigo $=7554336$

[3] Alfonso, A., Blanco, G.; Cedeño, J.; Suárez, P. (2019). Modelo de gestión para hoteles de categorías superiores en el destino Manta, Ecuador. Recuperdo http://eds.b.ebscohost.com/eds/pdfviewer/pdfviewer?vid=1\&sid=f5a023 42-454b-4837-b15f-671aab2d3dee\%40sessionmgr101

[4] Medina, A.; Nogueria, D.; Hernández, A.; Comas, R. (2019). Procedimiento para la gestión por procesos: métodos y herramientas de apoyo. Recuperado de https://scielo.conicyt.cl/scielo.php?pid=S071833052019000200328\&script=sci_arttext\&tlng=en

[5] Barrios, K.; Contreras, J.; Olivero, E. (2019). La Gestión por Procesos en las Pymes de Barranquilla: Factor Diferenciador de la Competitividad Organizacional. Recuperado https://scielo.conicyt.cl/scielo.php?pid=S071807642019000200103\&script=sci_arttext\&tlng=n

[6] Durarte, A.; Kiat, S.; Bressan,A.; O’Shea, M.; Sakellarios, N.; Koresis, A.; Buitragi, M.; Santoni, L. ( 2020). COVID-19, secuelas, impactos y empresas hoteleras: una perspectiva internacional. Recuperado de https://www.sciencedirect.com/science/article/pii/S0278431920302061

[7] Cepal. (2020). Medidas de recuperación del sector turístico en América Latina y el Caribe: una oportunidad para promover la sostenibilidad y la resiliencia. Recuperado de https://repositorio.cepal.org/bitstream/handle/11362/45770/1/S2000441_ es.pdf

[8] INEI (2020). Encuesta Mensual de Servicio Prestados a Empresa. Recuperado de https://www.inei.gob.pe/

[9] Otzen, T. \& Manterola, C. (2016). Técnicas de Muestreo sobre una Población a Obtenido de https://scielo.conicyt.cl/scielo.php?pid=S071795022017000100037\&scri $\mathrm{pt}=\mathrm{sci} \_$abstract

[10] Mallar, M. (2010). En Gestión por procesos: un enfoque de gestión eficiente. Visión de futuro, 13 (1). Recuperado de: http://www.redalyc.org/html/3579/357935475004/

[11] Salazar, A., Prado, H., Garro, L., Díaz, J., Ariaga, A. \& Uribe, Y. (2020). $\mathrm{La}$ incidencia de la gestión por procesos en los organismos gubernamentales. Gestión I+D, 5(1 Especial), 85-103.

[12] Rojas, Patricia (2001). ¿Qué es la competitividad? No. 9. IICA.

[13] Pérez, J. (2012). Gestión por procesos. Madrid: ESIC.

[14] González, María Antonia Morales, y José Luis Pech Várguez (2000). Competitividad y estrategia: el enfoque de las competencias esenciales y el enfoque basado en los recursos». (197): 17.

[15] Barraza, Bernardo Javier Sánchez (2006). LAS MYPES EN PERÚ. SU IMPORTANCIA Y PROPUESTA TRIBUTARIA. Quipukamayoc 13(25):127-31. doi: 10.15381/quipu.v13i25.5433.

[16] Ato, M., López-García, J. J., \& Benavente, A. (2013). Un sistema de clasificación de los diseños de investigación en psicología. Anales de Psicología/Annals of Psychology, 29(3), 1038-1059.

[17] Romero, K. (2019). La Gestión por Procesos y la generación de valor para el consumidor de las MYPES de Servicio de Restaurantes de Tacna 2019. (Tesis de Licenciatura). Universidad Privada de Tacna, Perú. Recuperado de: http://repositorio.upt.edu.pe/bitstream/UPT/957/1/Romero-CalderonMorelia.pdf

[18] Aragón, A., Rubio, A. \& Delgado, Serna, A. \& Chablé, J. (2010). Estrategia y competitividad empresarial: Un estudio en las MiPyMEs de Tabasco. En Investigación y Ciencia. [En línea]. Recuperado de http://www.redalyc.org/pdf/674/67413393002.pdf 